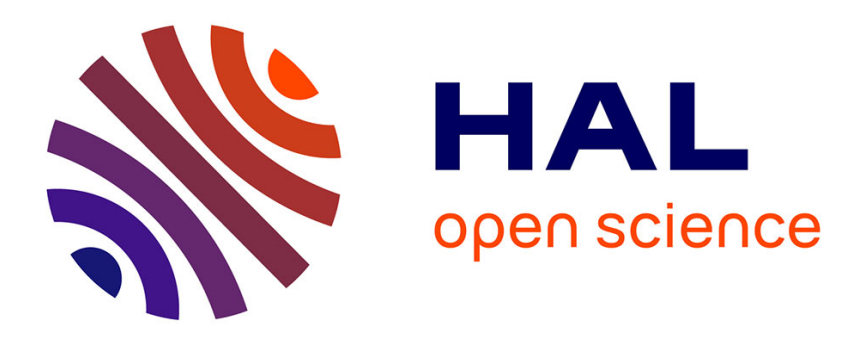

\title{
Global weak Solutions for the Landau-Lifschitz equation with magnetostriction
}

\author{
Gilles Carbou, M.A. Efendiev, P. Fabrie
}

\section{To cite this version:}

Gilles Carbou, M.A. Efendiev, P. Fabrie. Global weak Solutions for the Landau-Lifschitz equation with magnetostriction. Mathematical Methods in the Applied Sciences, 2011, 34 (10), pp.1274-1288. 10.1002/mma.1440 . hal-00868046

\section{HAL Id: hal-00868046 https://hal.science/hal-00868046}

Submitted on 28 May 2019

HAL is a multi-disciplinary open access archive for the deposit and dissemination of scientific research documents, whether they are published or not. The documents may come from teaching and research institutions in France or abroad, or from public or private research centers.
L'archive ouverte pluridisciplinaire HAL, est destinée au dépôt et à la diffusion de documents scientifiques de niveau recherche, publiés ou non, émanant des établissements d'enseignement et de recherche français ou étrangers, des laboratoires publics ou privés. 


\title{
Global weak solutions for the Landau-Lifschitz Equation with Magnetostriction
}

\author{
G. Carbou ${ }^{1}$, M. A. Efendiev² and P. Fabrie ${ }^{1}$ \\ ${ }^{1}$ Institut de Mathématiques de Bordeaux, UMR 5251, Université de Bordeaux, 351 cours de la Libération, 33405 \\ Talence cedex, France. \\ ${ }^{2}$ Center of Mathematical Sciences, GSF/Technical University Munich, Boltzmann Str. 3, 85747 Garching/Munich, \\ Germany.
}

Abstract. In this paper we prove the global in time existence for weak solutions to a Landau-Lifschitz system with magnetostriction arising from ferromagnetism theory. We describe also the $\omega$-limit set of a solution.

Keywords. Landau-Lifschitz equation, magnetostriction, weak solutions, ferromagnetism.

AMS classification. 35K55, 35Q55, 35Q74.

\section{Modelization}

The applications of ferromagnetic materials are more and more numerous: hard-disks, recording heads, ferromagnetic paints, etc. A general description of these materials is given by Landau-Lifschitz in [21] (see also [9], [18] and [24]). The ferromagnetic materials are spontaneously magnetized. Their magnetization is described by a vector field $m: \mathbb{R}^{+} \times \Omega \longrightarrow \mathbb{R}^{3}$ call the magnetic moment, where we denote by $\Omega$ the ferromagnetic domain. We assume that the material satisfies the saturation constraint:

$$
|m|=1
$$

The following Landau-Lifschitz equation describes the behaviour of $m$ :

$$
\frac{\partial m}{\partial t}=-m \wedge H_{e}-m \wedge\left(m \wedge H_{e}\right)
$$

In simplified models, the so called effective field $H_{e}$ is given by

$$
H_{e}=\Delta m+h_{d}(m)
$$

where the demagnetizing field $h_{d}(m)$ is deduced from $m$ by the static Maxwell equations together with the law of Faraday:

$$
\left\{\begin{array}{l}
\operatorname{div}\left(h_{d}(m)+\bar{m}\right)=0 \text { in } \mathbb{R}^{3}, \\
\operatorname{curl} h_{d}(m)=0 \text { in } \mathbb{R}^{3} .
\end{array}\right.
$$

In the previous system, $\bar{m}$ is the extension of $m$ by zero outside $\Omega$.

Existence results for weak solutions of (2) can be found in [23] or [12]. Existence of strong solutions is investigate in [13], [14] or [15] and [11] for a more complete model.

In this paper, we investigate the coupling of magnetic and mechanical effects by studying the complete Landau-Lifschitz equation with magnetostriction. In the following two subsections, we give a complete description of the model. Our main results are stated in subsection 1.3. Roughly speaking, we establish a global existence results for the weak solutions of the Landau-Lifschitz equation with magnetostriction, are we describe the $\omega$-limit set of a trajectory. 


\subsection{Landau-Lifschitz equation with magnetostriction}

In the physical litterature (see [21]) or in numerical studies (see [5]), the model for a ferromagnetic body with magnetostriction is the following. The magnetic moment satisfies the Landau-Lifschitz equation:

$$
\left\{\begin{array}{l}
\frac{\partial m}{\partial t}=-m \wedge H_{e f f}-m \wedge\left(m \wedge H_{e f f}\right) \text { on } \mathbb{R}^{+} \times \Omega \\
\text { with } H_{e f f}=\Delta m+h_{d}(m)+\Psi(m)+\left(\lambda^{\mathrm{m}}: \sigma\right) m \\
m(t=0)=m_{0} \text { in } \Omega \\
\partial_{\mathbf{n}} m=0 \text { on } \mathbb{R}^{+} \times \partial \Omega
\end{array}\right.
$$

where

- the initial data $m_{0}$ is supposed to be given in $H^{1}\left(\Omega ; S^{2}\right)$,

- $h_{d}(m)$ is the demagnetizing field,

- $\Psi$ is an anisotropic term. This term is the differential of a non negative quadratic form $\Phi: \mathbb{R}^{3} \longrightarrow \mathbb{R}$. Consequently it is a linear term,

- the magnetostriction field $h_{\mathrm{m}}$ links the magnetic moment $m$ with the stress tensor $\sigma$. It's given by

$$
h_{\mathrm{m}}=\left(\lambda^{\mathrm{m}}: \sigma\right) m,
$$

where $\lambda^{\mathrm{m}}$ is a symmetric non negative 4-tensor and $\sigma$ is the stress tensor. It is a 2-tensor (see below)

Remark 1 The usual notations and definitions about tensor calculus are recalled in Subsection 1.2.1.

In order to take into account the magnetostriction, the Landau-Lifschitz equation is coupled with the following wave equation:

$$
\left\{\begin{array}{l}
\frac{\partial^{2} u}{\partial t^{2}}-\operatorname{div} \sigma=0 \text { on } \mathbb{R}^{+} \times \Omega \\
u(t=0)=u_{0} \text { in } \Omega \\
\frac{\partial u}{\partial t}(t=0)=u_{1} \text { in } \Omega \\
u(t, x)=0 \text { on } \mathbb{R}^{+} \times \partial \Omega
\end{array}\right.
$$

where

- the stress tensor $\sigma$ satisfies $\sigma=\lambda^{\mathrm{e}}: \varepsilon^{\mathrm{e}}$, where $\lambda^{\mathrm{e}}$ is a symmetric positive 4-tensor,

- the tensor $\varepsilon^{\mathrm{e}}$ is obtained from the deformation tensor $\varepsilon(u)$ and the magnetic tensor $\varepsilon^{\mathrm{m}}$ by

$$
\varepsilon(u)=\varepsilon^{\mathrm{e}}+\varepsilon^{\mathrm{m}},
$$

- the deformation tensor $\varepsilon(u)$ is defined by $\varepsilon(u)_{i j}=\frac{1}{2}\left(\frac{\partial u^{i}}{\partial x_{j}}+\frac{\partial u^{j}}{\partial x_{i}}\right)$

- $\varepsilon^{\mathrm{m}}=\lambda^{\mathrm{m}}: m \otimes m$, that is $\varepsilon_{i j}^{\mathrm{m}}=\sum_{i j k l} \lambda_{i j k l}^{\mathrm{m}} m^{k} m^{l}$, 
The initial data $u_{0}$ is supposed to be in $H_{0}^{1}\left(\Omega ; \mathbb{R}^{3}\right)$ and $u_{1}$ is supposed to be in $L^{2}\left(\Omega ; \mathbb{R}^{3}\right)$.

We consider the Laudau-Lisfschitz-Gilbert form for the Landau-Lifschitz part of the system. In addition, we eliminate the variables $\sigma, \varepsilon^{\mathrm{m}}$ and $\varepsilon^{\mathrm{e}}$ so we deal with the following system coupling the Landau Lifschitz equation:

$$
\left\{\begin{array}{l}
\frac{\partial m}{\partial t}-m \wedge \frac{\partial m}{\partial t}=-2 m \wedge H_{\text {eff }} \text { on } \mathbb{R}^{+} \times \Omega, \\
H_{\text {eff }}=\Delta m+h_{d}(m)+\Psi(m)+\left(\lambda^{\mathrm{m}}:\left(\lambda^{\mathrm{e}}: \varepsilon(u)\right)\right) m-\left(\lambda^{\mathrm{m}}:\left(\lambda^{\mathrm{e}}:\left(\lambda^{\mathrm{m}}: m \otimes m\right)\right)\right) m,
\end{array}\right.
$$

together with the wave equation:

$$
\frac{\partial^{2} u}{\partial t^{2}}-\operatorname{div}\left(\lambda^{\mathrm{e}}: \varepsilon(u)\right)=-\operatorname{div}\left(\lambda^{\mathrm{e}}:\left(\lambda^{\mathrm{m}}: m \otimes m\right)\right) \text { on } \mathbb{R}^{+} \times \Omega,
$$

with the initial and boundary conditions:

$$
\left\{\begin{array}{l}
m(t=0)=m_{0} \text { in } \Omega, \\
u(t=0)=u_{0} \text { in } \Omega, \\
\frac{\partial u}{\partial t}(t=0)=u_{1} \text { in } \Omega, \\
\partial_{\mathbf{n}} m=0 \text { on } \mathbb{R}^{+} \times \partial \Omega, \\
u(t, x)=0 \text { on } \mathbb{R}^{+} \times \partial \Omega .
\end{array}\right.
$$

Remark 2 For regular enough solutions, the Landau-Lifschitz equation (3) is equivalent to tho LandauLifschitz-Gilbert equation (5). This last formulation is more convenient to write a weak formulation and to establish a global existence theorem.

\section{$1.2 \quad$ Structural properties}

\subsubsection{Tensor calculus}

Let us recall notations and definitions about tensors:

- let $\lambda$ be a 4 -tensor $\lambda=\left(\lambda_{i j k l}\right)$. We say that $\lambda$ is symmetric if

$$
\lambda_{i j k l}=\lambda_{j i k l}=\lambda_{i j l k}=\lambda_{k l i j} .
$$

- We say that a symmetric 4-tensor is positive if there exists a constant $\lambda^{*}$ such that:

$$
\forall \xi^{i j}, \sum_{i j k l} \lambda_{i j k l} \xi^{i j} \xi^{k l} \geq \lambda^{*} \sum_{i j}\left(\xi^{i j}\right)^{2}
$$

- If $\lambda$ is a 4 -tensor and $\nu$ is a two tensor, we denote by $\lambda: \nu$ the 2 -tensor given by

$$
(\lambda: \nu)_{i j}=\sum_{k l} \lambda_{i j k l} \nu_{k l} .
$$

- If $\mu$ and $\nu$ are two 2-tensors, then $\mu: \nu$ is a scalar given by

$$
\mu: \nu=\sum_{i j} \mu_{i j} \nu_{i j}
$$


- for $(\xi, \zeta) \in \mathbb{R}^{3} \times \mathbb{R}^{3}$, then $\xi \otimes \zeta$ is the 2 -tensor which entries are given by

$$
(\xi \otimes \xi)_{i j}=\xi^{i} \zeta^{j} .
$$

We state now useful lemmas concerning tensors.

Lemma 1 Let $\lambda$ be a symmetric 4-tensor, let $A$ be a symmetric two tensor, and let $\xi_{1}$ and $\xi_{2}$ in $\mathbb{R}^{3}$. We have

$$
(\lambda: A) \xi_{1} \cdot \xi_{2}=A:\left(\lambda: \xi_{1} \otimes \xi_{2}\right) .
$$

Proof: we prove this lemma by straightforward calculations.

We define $\mathcal{Q}$ by

$$
\left.\mathcal{Q}(m)=\left(\lambda^{\mathrm{e}}:\left(\lambda^{\mathrm{m}}: m \otimes m\right)\right)\right):\left(\lambda^{\mathrm{m}}: m \otimes m\right)
$$

Lemma 2 The map $\mathcal{Q}: \mathbb{R}^{3} \longrightarrow \mathbb{R}$ is $\mathcal{C}^{\infty}$ and

$$
\nabla \mathcal{Q}(m)=4\left(\lambda^{\mathrm{m}}:\left(\lambda^{\mathrm{e}}:\left(\lambda^{\mathrm{m}}: m \otimes m\right)\right)\right) m .
$$

Proof: this lemma is a simple consequence of Lemma 1

Lemma 3 Let $\lambda$ be a symmetric positive 4-tensor, let $A$ and $B$ be two 2-tensors. Then

$$
(\lambda: A): B \leq((\lambda: A): A)^{\frac{1}{2}}((\lambda: B): B)^{\frac{1}{2}} .
$$

Proof: we consider $\chi:\{1,2,3\}^{2} \longrightarrow\{1,2, \ldots, 9\}$ a bijective map. Let $\Lambda \in \mathcal{M}_{9}(\mathbb{R})$ the matrix of entries $\Lambda_{\chi(i, j) \chi(k, l)}=\lambda_{i j k l}$. In the same way, we consider $\bar{A} \in \mathbb{R}^{9}$ such that $\bar{A}_{\chi(i, j)}=A_{i j}$, and $\bar{B} \in \mathbb{R}^{9}$ such that $\bar{B}_{\chi(i, j)}=B_{i j}$.

We have $(\lambda: A): B=\Lambda \bar{A} \cdot \bar{B}$.

The matrix $\Lambda$ is symmetric positive, by property of $\lambda$ and we introduce $\Gamma \in \mathcal{M}_{9}(\mathbb{R})$ the square root of $\Lambda$. We have:

$$
(\lambda: A): B=\Gamma \bar{A} \cdot \Gamma \bar{B} \leq\|\Gamma \bar{A}\|\|\Gamma \bar{B}\|
$$

by Cauchy Schwartz inequality. Now

$$
\|\Gamma \bar{A}\|^{2}=\Gamma \bar{A} \cdot \Gamma \bar{A}=\Lambda \bar{A} \cdot \bar{A}=(\lambda: A): A,
$$

and in the same way

$$
\|\Gamma \bar{B}\|^{2}=(\lambda: B): B
$$

and the proof of Lemma 3 is complete.

\subsubsection{Energy Formula}

The calculations in this section are formal. They are valid for regular enough solutions.

First, taking the scalar product of (5) with $m$, we obtain that $\frac{\partial m}{\partial t} \cdot m=0$, that is $\frac{\partial}{\partial t}\left(|m|^{2}\right)=0$. Since the initial data satisfies $\left|m_{0}\right|=1$, then for all time, $m$ satisfies the saturation constraint $|m|=1$.

The proof of the existence of solutions for (5)-(6)-(7) is built on energy estimates which are the consequence of algebraic properties. Formally, for regular enough functions, the following calculations hold:

On one hand, we take the inner product of (5) by $\frac{\partial m}{\partial t}-2 H_{e f f}$, so that we obtain that

$$
\int_{\Omega} \frac{\partial m}{\partial t} \cdot\left(\frac{\partial m}{\partial t}-2 H_{e f f}\right)=0 .
$$

From the first three terms of the effective field, we have: 
- by integrations by parts:

$$
\int_{\Omega} \Delta m \cdot \frac{\partial m}{\partial t}=-\frac{1}{2} \frac{d}{d t} \int_{\Omega}|\nabla m|^{2}
$$

- since $-h_{d}$ is an orthogonal projector for the $L^{2}$ inner product,

$$
\int_{\Omega} h_{d}(m) \cdot \frac{\partial m}{\partial t}=-\frac{1}{2} \frac{d}{d t} \int_{\mathbb{R}^{3}}\left|h_{d}(m)\right|^{2},
$$

- since $\Psi=-\nabla \Phi$,

$$
\int_{\Omega} \Psi(m) \cdot \frac{\partial m}{\partial t}=-\frac{1}{2} \frac{d}{d t} \int_{\Omega} \Phi(m) .
$$

For the magnetostriction terms, we first remark that by symmetry of the 4-tensor $\lambda^{\mathrm{m}}$ and by Lemma 2 , we have

$$
2 \int_{\Omega}\left(\lambda^{\mathrm{m}}:\left(\lambda^{\mathrm{e}}:\left(\lambda^{\mathrm{m}}: m \otimes m\right)\right)\right) m \cdot \frac{\partial m}{\partial t}=\frac{1}{2} \frac{d}{d t} \int_{\Omega} \mathcal{Q}(m)
$$

where $\mathcal{Q}(m)$ is a non negative term of fourth order:

$$
\left.\mathcal{Q}(m)=\left(\lambda^{\mathrm{e}}:\left(\lambda^{\mathrm{m}}: m \otimes m\right)\right)\right):\left(\lambda^{\mathrm{m}}: m \otimes m\right) .
$$

In addition, by Lemma 1, we have

$$
\begin{aligned}
\left.2 \int_{\Omega}\left(\lambda^{\mathrm{m}}:\left(\lambda^{\mathrm{e}}: \varepsilon(u)\right)\right)\right) m \cdot \frac{\partial m}{\partial t} & =2 \int_{\Omega} \varepsilon(u):\left(\lambda^{\mathrm{e}}:\left(\lambda^{\mathrm{m}}: m \otimes \frac{\partial m}{\partial t}\right)\right) \\
& =\int_{\Omega} \varepsilon(u): \frac{\partial}{\partial t}\left(\lambda^{\mathrm{e}}:\left(\lambda^{\mathrm{m}}: m \otimes m\right)\right) .
\end{aligned}
$$

So, from (5) we obtain that

$$
\begin{aligned}
& \int_{\Omega}\left|\frac{\partial m}{\partial t}\right|^{2}+\frac{d}{d t}\left[\int_{\Omega}\left(|\nabla m|^{2}+\Phi(m)\right)+\int_{\mathbb{R}^{3}}\left|h_{d}(m)\right|^{2}+\frac{1}{2} \int_{\Omega} \mathcal{Q}(m)\right] \\
& -\int_{\Omega} \varepsilon(u): \frac{\partial}{\partial t}\left(\lambda^{\mathrm{e}}:\left(\lambda^{\mathrm{m}}: m \otimes m\right)\right)=0 .
\end{aligned}
$$

On the other hand, we take the inner product of the second equation in (6) by $\frac{\partial u}{\partial t}$.

Using that $\lambda^{\mathrm{e}}$ is symmetric, and that $u=0$ on $\partial \Omega$, we obtain after integration by parts that:

$$
-\int_{\Omega} \operatorname{div}\left(\lambda^{\mathrm{e}}: \varepsilon(u)\right) \cdot \frac{\partial u}{\partial t}=\frac{1}{2} \frac{d}{d t} \int_{\Omega}\left(\lambda^{\mathrm{e}}: \varepsilon(u)\right): \varepsilon(u) .
$$

Furthermore, by integration by parts,

$$
\left.\left.\int_{\Omega}-\operatorname{div}\left(\lambda^{\mathrm{e}}:\left(\lambda^{\mathrm{m}}: m \otimes m\right)\right)\right] \cdot \frac{\partial u}{\partial t}=\int_{\Omega}\left(\lambda^{\mathrm{e}}:\left(\lambda^{\mathrm{m}}: m \otimes m\right)\right)\right): \frac{\partial \varepsilon(u)}{\partial t}
$$

by symmetry of $\lambda^{\mathrm{e}}$.

Hence we obtain from (6) that

$$
\frac{1}{2} \frac{d}{d t}\left[\int_{\Omega}\left|\frac{\partial u}{\partial t}\right|^{2}+\int_{\Omega}\left(\lambda^{\mathrm{e}}: \varepsilon(u)\right): \varepsilon(u)\right]=\int_{\Omega}\left(\lambda^{\mathrm{e}}:\left(\lambda^{\mathrm{m}}: m \otimes m\right)\right): \frac{\partial \varepsilon(u)}{\partial t} .
$$

Adding up (8) and (9), we obtain the energy formula:

$$
\frac{d}{d t} \mathcal{E}(t)+\int_{\Omega}\left|\frac{\partial m}{\partial t}\right|^{2}=0
$$


with

$$
\begin{aligned}
\mathcal{E}(t)= & \int_{\Omega}\left[|\nabla m|^{2}+\left|h_{d}(m)\right|^{2}+\Phi(m)\right] \\
& \left.+\frac{1}{2} \int_{\Omega}\left[\left(\lambda^{\mathrm{e}}:\left(\lambda^{\mathrm{m}}: m \otimes m\right)\right)\right):\left(\lambda^{\mathrm{m}}: m \otimes m\right)-2 \varepsilon(u):\left(\lambda^{\mathrm{e}}:\left(\lambda^{\mathrm{m}}: m \otimes m\right)\right)\right] \\
& +\frac{1}{2} \int_{\Omega}\left[\left(\lambda^{\mathrm{e}}: \varepsilon(u)\right): \varepsilon(u)+\left|\frac{\partial u}{\partial t}\right|^{2}\right] .
\end{aligned}
$$

Remark 3 Because of the positiveness of $\lambda^{e}$, in the energy, $\mathcal{Q}(m)$ and $\left(\lambda^{e}: \varepsilon(u)\right): \varepsilon(u)$ are positive. The bad sign term $-2 \varepsilon(u):\left(\lambda^{e}:\left(\lambda^{m}: m \otimes m\right)\right)$ can be balanced by both good sign terms, since applying Lemma 3 with $\lambda=\lambda^{e}, A=\varepsilon$ and $B=\lambda^{m}: m \otimes m$., we have:

$$
\varepsilon:\left(\lambda^{e}:\left(\lambda^{m}: m \otimes m\right)\right) \leq \mathcal{Q}(m)^{\frac{1}{2}} \cdot\left(\left(\lambda^{e}: \varepsilon\right): \varepsilon\right)^{\frac{1}{2}} .
$$

On the contrary, the part of the energy coming from the magnetostriction terms is non coercive since it does not control the term $\|\nabla u\|_{L^{2}(\Omega)}^{2}$ if we simply apply Young inequality to balance the bad sign term.

\subsection{Statement of the results}

Definition 1 We say that $(m, u)$ is a weak solution for (5)-(6)-(7) if

1. $m \in L^{\infty}\left(\mathbb{R}^{+} ; H^{1}\left(\Omega ; \mathbb{R}^{3}\right)\right)$ satisfies the saturation constraint $|m(t, x)|=1$ for almost every $(t, x) \in$ $\mathbb{R}^{+} \times \Omega$,

2. $\frac{\partial m}{\partial t} \in L^{2}\left(\mathbb{R}^{+} ; L^{2}\left(\Omega ; \mathbb{R}^{3}\right)\right)$,

3. $m(0, \cdot)=m_{0}$ in the trace sense in $H^{\frac{1}{2}}(\Omega)$,

4. $u \in L^{\infty}\left(\mathbb{R}^{+} ; H_{0}^{1}\left(\Omega ; \mathbb{R}^{3}\right)\right)$ and $\frac{\partial u}{\partial t} \in L^{\infty}\left(\mathbb{R}^{+} ; L^{2}\left(\mathbb{R}^{3} ; \mathbb{R}^{3}\right)\right)$,

5. $u(0, \cdot)=u_{0}$ in the trace sense in $H^{\frac{1}{2}}(\Omega)$,

6. for all $\chi \in \mathcal{C}_{c}^{\infty}\left(\mathbb{R}^{+} ; H^{1}\left(\Omega ; \mathbb{R}^{3}\right)\right)$

$$
\begin{array}{r}
\int_{\mathbb{R}^{+} \times \Omega}\left(\frac{\partial m}{\partial t}-m \wedge \frac{\partial m}{\partial t}\right) \chi(t, x) d t d x=2 \int_{\mathbb{R}^{+} \times \Omega} \sum_{i=1}^{3} m \wedge \frac{\partial m}{\partial x_{i}} \cdot \frac{\partial \chi}{\partial x_{i}}-2 \int_{\Omega}\left(h_{d}(m)+\Psi(m)\right) \cdot \chi \\
-2 \int_{\Omega}\left(\left(\lambda^{m}:\left(\lambda^{e}: \varepsilon(u)\right)\right) m-\left(\lambda^{m}:\left(\lambda^{e}:\left(\lambda^{m}: m \otimes m\right)\right)\right) m\right) \cdot \chi,
\end{array}
$$

7. for all $\chi \in \mathcal{C}_{c}^{\infty}\left(\mathbb{R}^{+} ; H_{0}^{1}\left(\Omega ; \mathbb{R}^{3}\right)\right)$

$$
\int_{\mathbb{R}^{+} \times \Omega} \frac{\partial u}{\partial t} \cdot \frac{\partial \chi}{\partial t}-\int_{\mathbb{R}^{+} \times \Omega}\left(\lambda^{e}: \varepsilon(u)\right): \varepsilon(\chi)+\int_{\Omega} u_{1} \chi(0, x) d x=-\int_{\mathbb{R}^{+} \times \Omega}\left(\lambda^{e}:\left(\lambda^{m}: m \otimes m\right)\right): \varepsilon(\chi),
$$

8. for all $t \geq 0$, we have the following energy inequality:

$$
\mathcal{E}(t)+\int_{0}^{t} \int_{\Omega}\left|\frac{\partial m}{\partial t}\right|^{2} \leq \mathcal{E}(0)
$$

where $\mathcal{E}$ is defined by (11). 
Remark 4 Since $m \in L^{\infty}\left(0, T ; H^{1}(\Omega)\right)$ and $\frac{\partial m}{\partial t} \in L^{2}\left(0, T ; L^{2}(\Omega)\right)$, then $m \in \mathcal{C}^{0}\left(0, T ; H^{\frac{1}{2}}(\Omega)\right)$ (see [22]) and $m \in \mathcal{C}^{0}\left(0, T ; H_{w}^{1}(\Omega)\right)$ (see [8] Lemma II.5.9). So the trace of $m$ at $t=0$ exists in $H^{\frac{1}{2}}(\Omega)$ for example. In the same way, $u \in \mathcal{C}^{0}\left(0, T ; H^{\frac{1}{2}}(\Omega)\right)$. Moreover, $u \in L^{\infty}\left(0, T ; H_{0}^{1}(\Omega)\right.$ so $\operatorname{div}\left(\lambda^{e}: \varepsilon(u)\right)$ is in $L^{\infty}\left(0, T ; H^{-1}\right)$, and by the wave equation, $\frac{\partial^{2} u}{\partial t^{2}} \in L^{\infty}\left(0, T ; H^{-1}(\Omega)\right)$. So $\frac{\partial u}{\partial t} \in \mathcal{C}^{0}\left(0, T ; H^{-\frac{1}{2}}(\Omega) \cap\right.$ $\mathcal{C}^{0}\left(0, T ; L_{w}^{2}(\Omega)\right)$. Hence the trace of $\frac{\partial u}{\partial t}$ at $t=0$ as a sense in $H^{-\frac{1}{2}}(\Omega)$.

Our first theorem is an existence result for global in time weak solutions of the system (5)-(6)-(7).

Theorem 1 Let $m_{0} \in H^{1}\left(\Omega ; S^{2}\right)$, let $u_{0} \in H_{0}^{1}\left(\Omega ; \mathbb{R}^{3}\right)$ and $u_{1} \in L^{2}\left(\Omega ; \mathbb{R}^{3}\right)$. Then there exists a weak solution for (5)-(6)-(7).

Remark 5 The formal calculations of the previous section are not allowed for weak solutions. Therefore, the saturation constraint and the energy inequality are obtained by construction. We remark that we only obtain an inequality energy (and not an equality as in the formal calculations). This is usual for the weak solutions of the Landau-Lifschitz equations (see [2] and [12]).

Our second result describes the $\omega$-limit set of a fixed solution fo (5)-(6)-(7).

Definition 2 Let $m$ be a weak solution of (5)-(6)-(7) given by Theorem 1. Let $m_{\infty} \in H^{1}(\Omega)$. We say that $m_{\infty}$ is in the $\omega$-limit set of $m$ if there exists a sequence of times $\left(t_{n}\right)_{n \in \mathbb{N}}$ such that $t_{n}$ tends to $+\infty$ and $m\left(t_{n}\right)$ tends weakly to $m_{\infty}$ in $H^{1}(\Omega)$ when $n$ tends to $+\infty$.

Theorem 2 Let $m$ be a weak solution of (5)-(6)-(7). Its $\omega$-limit set is non empty, and if $m_{\infty}$ is in the $\omega$-limit set of $m$, then $m_{\infty}$ satisfies the saturation constraint $\left|m_{\infty}\right|=1$ and satisfies, for all test function $\xi \in H^{1}\left(\Omega ; \mathbb{R}^{3}\right)$,

$$
\begin{aligned}
-\int_{\Omega} \sum_{i=1}^{3} m_{\infty} \wedge \frac{\partial m_{\infty}}{\partial x_{i}} \cdot \frac{\partial \xi}{\partial x_{i}}+\int_{\Omega} & \left(h_{d}\left(m_{\infty}\right)+\psi\left(m_{\infty}\right)+\left(\lambda^{m}:\left(\lambda^{e}: \varepsilon\left(u_{\infty}\right)\right)\right) m_{\infty}\right) \cdot \xi \\
& -\int_{\Omega}\left(\left(\lambda^{m}:\left(\lambda^{e}:\left(\lambda^{m}: m_{\infty} \otimes m_{\infty}\right)\right)\right) m_{\infty}\right) \cdot \xi=0 .
\end{aligned}
$$

where $u_{\infty}$ is deduced from $m_{\infty}$ by:

$$
\left\{\begin{array}{l}
u_{\infty} \in H_{0}^{1}(\Omega) \\
\operatorname{div}\left(\lambda^{e}: \varepsilon\left(u_{\infty}\right)\right)=\operatorname{div}\left(\lambda^{e}:\left(\lambda^{m}: m_{\infty} \otimes m_{\infty}\right)\right) \text { in } H^{-1}(\Omega) .
\end{array}\right.
$$

The paper is organised as follows. In the following subsection, we recall the Aubin-Simon compacteness lemma. Theorem 1 is proved in Sections 2 and 3. Theorem 2 is established in Section 4.

Our proof of Theorem 1 follows the method due to Alouges and Soyeur in [2] and generalized in [12] for the system coupling the Landau-Lifschitz with the Maxwell equations. First we study a penalized system in which the saturation constraint is relaxed and we take the limit when the penalization constant tends to zero. The new difficulty here is that the energy coming from the magnetostriction is non coercive (see Remark 3). The lack of coercivity is balanced by coupling the magnetostriction part with the penalization term (see Section 2.3).

Concerning the description of the $\omega$-limit set, the key tool is taking averages for $m$ and $u$ on time intervals $\left[t_{n}-a, t_{n}+a\right]$, and performing the limit when $n$ tends to $+\infty$ in a first step and when $a$ tends to $+\infty$ in a second step. This method is used in [12] for a simpler model.

Remark 6 Ferromagnetism is a wide domain in Physics. In Mathematics, recent developments have been obtained from the numerical point of view (see [6], [7], [19], [20] for example). Asymptotic studies are done in [1], [4], [10], [17] for example. In particular, the description of wall structures is a very important and challenging question (see [10] and [16] and the references therein). The interested reader can also read [3] for a related model of ferroelectric materials. 


\subsection{Compactness lemma}

By applying the Aubin-Simon lemma (see [8] Theorem II.5.16), we obtain:

Lemma 4 We define $W$ by

$$
W=\left\{v \in L^{\infty}\left(0, T ; H^{1}(\Omega)\right), \frac{\partial v}{\partial t} \in L^{2}\left(0, T ; L^{2}(\Omega)\right)\right\} .
$$

Then the injection of $W$ in $L^{\infty}\left(0, T ; L^{4}(\Omega)\right)$ is compact.

\section{Penalized system}

We consider for $\eta>0$ the following penalized system:

$$
\left\{\begin{array}{l}
\frac{\partial m^{\eta}}{\partial t}+m^{\eta} \wedge \frac{\partial m^{\eta}}{\partial t}-2 H_{e f f}^{\eta}+\frac{1}{\eta}\left(\left|m^{\eta}\right|^{2}-1\right) m^{\eta}=0 \text { on } \mathbb{R}^{+} \times \Omega, \\
H_{e f f}^{\eta}=\Delta m^{\eta}+h_{d}\left(m^{\eta}\right)+\Psi\left(m^{\eta}\right)+\left(\lambda^{\mathrm{m}}:\left(\lambda^{\mathrm{e}}: \varepsilon\left(u^{\eta}\right)\right)\right) m^{\eta}-\left(\lambda^{\mathrm{m}}:\left(\lambda^{\mathrm{e}}:\left(\lambda^{\mathrm{m}}: m^{\eta} \otimes m^{\eta}\right)\right)\right) m^{\eta} \\
\frac{\partial^{2} u^{\eta}}{\partial t^{2}}-\operatorname{div}\left(\lambda^{\mathrm{e}}: \varepsilon\left(u^{\eta}\right)\right)=-\operatorname{div}\left(\lambda^{\mathrm{e}}:\left(\lambda^{\mathrm{m}}: m^{\eta} \otimes m^{\eta}\right)\right) \text { on } \mathbb{R}^{+} \times \Omega, \\
m^{\eta}(t=0)=m_{0} \\
u^{\eta}(t=0)=u_{0} \\
\frac{\partial u^{\eta}}{\partial t}(t=0)=u_{1} \\
\partial_{\mathbf{n}} m^{\eta}=0 \text { on } \mathbb{R}^{+} \times \partial \Omega \\
u^{\eta}(t, x)=0 \text { on } \mathbb{R}^{+} \times \partial \Omega
\end{array}\right.
$$

Claim : there exists a weak global in time solution for (12).

In this section, $\eta>0$ is fixed.

\subsection{First step: Galerkin approximation}

For $m$, we use an Galerkin basis $\left(e_{1}, e_{2}, \ldots\right)$ of eigenvectors of $-\Delta$ with homogeneous Neumann conditions at the boundary.

$$
\left\{\begin{array}{l}
-\Delta e_{i}=\alpha_{i} e_{i} \text { in } \Omega, \\
\partial_{\mathbf{n}} e_{i}=0 \text { on } \partial \Omega .
\end{array}\right.
$$

We denote by $V_{N}=\operatorname{span}\left(e_{1}, \ldots, e_{N}\right)$ and by $P_{N}$ the orthogonal projection map onto $V_{N}$.

For $u$, we use the Galerkin basis $\left(f_{1}, f_{2}, \ldots\right)$ of eigenvectors of $-\operatorname{div}\left(\lambda^{\mathrm{e}}: \varepsilon\right)$ with homogeneous Dirichlet conditions at the boundary:

$$
\left\{\begin{array}{l}
-\operatorname{div}\left(\lambda^{\mathrm{e}}: \varepsilon\left(f_{j}\right)\right)=\beta_{j} f_{j} \text { in } \Omega \\
f_{j}=0 \text { on } \partial \Omega
\end{array}\right.
$$

We denote by $W_{N}=\operatorname{span}\left(f_{1}, \ldots, f_{N}\right)$ and by $\Pi_{N}$ the orthogonal projection map onto $W_{N}$.

We consider for a fixed $N$ the solution $\left(m_{N}^{\eta}, u_{N}^{\eta}\right): \mathbb{R}_{t}^{+} \longrightarrow V_{N} \times W_{N}$ of the o.d.e. approximation: 


$$
\left\{\begin{array}{l}
\frac{\partial m_{N}^{\eta}}{\partial t}+P_{N}\left(m_{N}^{\eta} \wedge \frac{\partial m_{N}^{\eta}}{\partial t}\right)-2 P_{N}\left(H_{e f f}^{N}\right)+\frac{1}{\eta} P_{N}\left(\left(\left|m_{N}^{\eta}\right|^{2}-1\right) m_{N}^{\eta}\right)=0 \text { on } \mathbb{R}^{+} \\
H_{e f f}^{N}=\Delta m_{N}^{\eta}+h_{d}\left(m_{N}^{\eta}\right)+\Psi\left(m_{N}^{\eta}\right)+\left(\lambda^{\mathrm{m}}:\left(\lambda^{\mathrm{e}}: \varepsilon\left(u_{N}^{\eta}\right)\right)\right) m_{N}^{\eta}-\left(\lambda^{\mathrm{m}}:\left(\lambda^{\mathrm{e}}:\left(\lambda^{\mathrm{m}}: m_{N}^{\eta} \otimes m_{N}^{\eta}\right)\right)\right) m_{N}^{\eta} \\
\frac{\partial^{2} u_{N}^{\eta}}{\partial t^{2}}-\operatorname{div}\left(\lambda^{\mathrm{e}}: \varepsilon\left(u_{N}^{\eta}\right)\right)=-\Pi_{N}\left(\operatorname{div}\left(\lambda^{\mathrm{e}}:\left(\lambda^{\mathrm{m}}: m_{N}^{\eta} \otimes m_{N}^{\eta}\right)\right)\right) \text { on } \mathbb{R}^{+}, \\
m_{N}^{\eta}(t=0)=P_{N}\left(m_{0}\right) \\
u_{N}^{\eta}(t=0)=\Pi_{N}\left(u_{0}\right) \\
\frac{\partial u_{N}^{\eta}}{\partial t}(t=0)=\Pi_{N}\left(u_{1}\right)
\end{array}\right.
$$

In order to apply the Cauchy-Lipschitz theorem for this system, we remark that the operator $G^{N}(13)$ defined for $m_{N} \in V_{N}$ by

$$
\begin{aligned}
G^{N}\left(m_{N}\right): \quad V_{N} & \longrightarrow V_{N} \\
w & \mapsto w+P_{N}\left(m_{N} \wedge w\right)
\end{aligned}
$$

is invertible. Indeed, for a fixed $m_{N} \in V_{N}$, the operator $G^{N}\left(m_{N}\right)$ is linear on the finite dimensional space $V_{N}$. Its kernel is reduced to zero: if $G^{N}\left(m_{N}\right)(w)=0$, then $w=0$. Indeed, taking the inner product is $L^{2}(\Omega)$ with $w \in V_{N}$, we obtain

$$
\begin{aligned}
0 & =\int_{\Omega} G^{N}\left(m_{N}\right)(w) \cdot w \\
& =\int_{\Omega}|w|^{2}+\int_{\Omega} P_{N}\left(m_{N} \wedge w\right) \cdot w \\
& =\int_{\Omega}|w|^{2}+\int_{\Omega}\left(m_{N} \wedge w\right) \cdot w \\
& \text { since } P_{N} \text { is selfadjoint and since } w \in V_{N} \\
& =\int_{\Omega}|w|^{2}
\end{aligned}
$$

So, inverting this operator, the first equation can be written as

$$
\frac{\partial m_{N}^{\eta}}{\partial t}=F\left(m_{N}^{\eta}, u_{N}^{\eta}\right)
$$

which is an ordinary differential equation. Then by the Cauchy-Lipschitz theorem, there exists a unique solution for (13) which maximal existence time is denoted by $T_{N}$.

\subsection{Energy estimate on the Galerkin approximation}

On one hand, we take the inner product of the second equation in (13) by $\frac{\partial u_{N}^{\eta}}{\partial t}$.

Using that $\lambda^{\mathrm{e}}$ is symmetric, and that $u=0$ on $\partial \Omega$, we have:

$$
-\int_{\Omega} \operatorname{div}\left(\lambda^{\mathrm{e}}: \varepsilon\left(u_{N}^{\eta}\right)\right) \cdot \frac{\partial u_{N}^{\eta}}{\partial t}=\frac{1}{2} \frac{d}{d t} \int_{\Omega}\left(\lambda^{\mathrm{e}}: \varepsilon\left(u_{N}^{\eta}\right)\right): \varepsilon\left(u_{N}^{\eta}\right) .
$$


Furthermore, as $\frac{\partial u_{N}^{\eta}}{\partial t}$ belongs to $W_{N}$, one obtains

$$
\begin{aligned}
\int_{\Omega}-\Pi_{N}\left(\operatorname{div}\left(\lambda^{\mathrm{e}}:\left(\lambda^{\mathrm{m}}: m_{N}^{\eta} \otimes m_{N}^{\eta}\right)\right)\right) \cdot \frac{\partial u_{N}^{\eta}}{\partial t} & =\int_{\Omega}-\operatorname{div}\left(\lambda^{\mathrm{e}}:\left(\lambda^{\mathrm{m}}: m_{N}^{\eta} \otimes m_{N}^{\eta}\right)\right) \cdot \frac{\partial u_{N}^{\eta}}{\partial t} \\
& =\int_{\Omega}\left(\lambda^{\mathrm{e}}:\left(\lambda^{\mathrm{m}}: m_{N}^{\eta} \otimes m_{N}^{\eta}\right)\right): \frac{\partial \varepsilon\left(u_{N}^{\eta}\right)}{\partial t}
\end{aligned}
$$

by symmetry of $\lambda^{\mathrm{e}}$.

Hence we obtain that

$$
\frac{1}{2} \frac{d}{d t}\left(\int_{\Omega}\left|\frac{\partial u_{N}^{\eta}}{\partial t}\right|^{2}+\int_{\Omega}\left(\lambda^{\mathrm{e}}: \varepsilon\left(u_{N}^{\eta}\right)\right): \varepsilon\left(u_{N}^{\eta}\right)\right)=\int_{\Omega}\left(\lambda^{\mathrm{e}}:\left(\lambda^{\mathrm{m}}: m_{N}^{\eta} \otimes m_{N}^{\eta}\right)\right): \frac{\partial \varepsilon\left(u_{N}^{\eta}\right)}{\partial t} .
$$

On the other hand we take the inner product of the first equation in (13) by $\frac{\partial m_{N}^{\eta}}{\partial t}$. Since $\frac{\partial m_{N}^{\eta}}{\partial t}$ is in $V_{N}$ (so that we can remove $P_{N}$ ) we get

$$
\begin{aligned}
& \int_{\Omega}\left|\frac{\partial m_{N}^{\eta}}{\partial t}\right|^{2}+\frac{d}{d t} \int_{\Omega}\left(\left|\nabla m_{N}^{\eta}\right|^{2}+\Phi\left(m_{N}^{\eta}\right)+\frac{1}{2 \eta}\left(\left|m_{N}^{\eta}\right|^{2}-1\right)^{2}\right)+\frac{d}{d t} \int_{\mathbb{R}^{3}}\left|h_{d}\left(m_{N}^{\eta}\right)\right|^{2} \\
& -2 \int_{\Omega}\left(\lambda^{\mathrm{m}}:\left(\lambda^{\mathrm{e}}: \varepsilon\left(u_{N}^{\eta}\right)\right)\right) m_{N}^{\eta} \cdot \frac{\partial m_{N}^{\eta}}{\partial t}+2 \int_{\Omega}\left(\lambda^{\mathrm{m}}:\left(\lambda^{\mathrm{e}}:\left(\lambda^{\mathrm{m}}: m_{N}^{\eta} \otimes m_{N}^{\eta}\right)\right)\right) m_{N}^{\eta} \cdot \frac{\partial m_{N}^{\eta}}{\partial t}=0 .
\end{aligned}
$$

As in the formal case, we remark that by symmetry of the 4 -tensor $\lambda^{\mathrm{m}}$, we have

$$
2 \int_{\Omega}\left(\lambda^{\mathrm{m}}:\left(\lambda^{\mathrm{e}}:\left(\lambda^{\mathrm{m}}: m_{N}^{\eta} \otimes m_{N}^{\eta}\right)\right)\right) m_{N}^{\eta} \cdot \frac{\partial m_{N}^{\eta}}{\partial t}=\frac{1}{2} \frac{d}{d t} \int_{\Omega} \mathcal{Q}\left(m_{N}^{\eta}\right)
$$

with

$$
\left.Q(m)=\left(\lambda^{\mathrm{e}}:\left(\lambda^{\mathrm{m}}: m \otimes m\right)\right)\right):\left(\lambda^{\mathrm{m}}: m \otimes m\right) .
$$

In addition, by Lemma 1, we have

$$
\begin{aligned}
2 \int_{\Omega}\left(\lambda^{\mathrm{m}}:\left(\lambda^{\mathrm{e}}: \varepsilon\left(u_{N}^{\eta}\right)\right)\right) m_{N}^{\eta} \cdot \frac{\partial m_{N}^{\eta}}{\partial t} & =2 \int_{\Omega} \varepsilon\left(u_{N}^{\eta}\right):\left(\lambda^{\mathrm{e}}:\left(\lambda^{\mathrm{m}}: m_{N}^{\eta} \otimes \frac{\partial m_{N}^{\eta}}{\partial t}\right)\right) \\
& =\int_{\Omega} \varepsilon\left(u_{N}^{\eta}\right): \frac{\partial}{\partial t}\left(\lambda^{\mathrm{e}}:\left(\lambda^{\mathrm{m}}: m_{N}^{\eta} \otimes m_{N}^{\eta}\right)\right) .
\end{aligned}
$$

The previous three formulae together with (14) yield

$$
\int_{\Omega}\left|\frac{\partial m_{N}^{\eta}}{\partial t}\right|^{2}+\frac{d}{d t} \mathcal{E}_{N}^{\eta}+\frac{1}{2 \eta} \frac{d}{d t} \int_{\Omega}\left(\left|m_{N}^{\eta}\right|^{2}-1\right)^{2}=0
$$

where

$$
\begin{aligned}
\mathcal{E}_{N}^{\eta}(t)= & \int_{\Omega}\left(\left|\nabla m_{N}^{\eta}\right|^{2}+\Phi\left(m_{N}^{\eta}\right)\right)+\int_{\mathbb{R}^{3}}\left|h_{d}\left(m_{N}^{\eta}\right)\right|^{2} \\
& +\frac{1}{2} \int_{\Omega}\left(\mathcal{Q}\left(m_{N}^{\eta}\right)-2 \varepsilon\left(u_{N}^{\eta}\right):\left(\lambda^{\mathrm{e}}:\left(\lambda^{\mathrm{m}}: m_{N}^{\eta} \otimes m_{N}^{\eta}\right)\right)+\left|\frac{\partial u_{N}^{\eta}}{\partial t}\right|^{2}+\left(\lambda^{\mathrm{e}}: \varepsilon\left(u_{N}^{\eta}\right)\right): \varepsilon\left(u_{N}^{\eta}\right)\right) .
\end{aligned}
$$

We integrate this inequality with respect to time and we obtain: for all $T<T_{N}$,

$$
\mathcal{E}_{N}^{\eta}(T)+\frac{1}{2 \eta} \int_{\Omega}\left(\left|m_{N}^{\eta}\right|^{2}-1\right)^{2}+\int_{0}^{T} \int_{\Omega}\left|\frac{\partial m_{N}^{\eta}}{\partial t}\right|^{2}=\mathcal{E}_{N}^{\eta}(0)+\frac{1}{2 \eta} \int_{\Omega}\left(\left|P_{N}\left(m_{0}\right)\right|^{2}-1\right)^{2} .
$$

By Lemma 3, we obtain that the energy is positive, but if we use a simple Young inequality to absorb the bad sign term with the good sign terms given by the lemma, then we loose the control for the $L^{2}$ 
norm of $\nabla u_{N}^{\eta}$. To avoid this problem, we will absorb at this step the bad sign term using a part of the penalization term as it is explained below. With Lemma 3, there exists $C$ such that

$$
\varepsilon\left(u_{N}^{\eta}\right):\left(\lambda^{\mathrm{e}}:\left(\lambda^{\mathrm{m}}: m_{N}^{\eta} \otimes m_{N}^{\eta}\right)\right) \leq \frac{1}{4}\left(\lambda^{\mathrm{e}}: \varepsilon\left(u_{N}^{\eta}\right)\right): \varepsilon\left(u_{N}^{\eta}\right)+C\left\|m_{N}^{\eta}\right\|_{L^{4}}^{4} .
$$

Inequality (15) together with the claim give a uniform bound on $m_{N}^{\eta}$ and $u_{N}^{\eta}$ since

$$
\begin{aligned}
\mathcal{E}_{N}^{\eta}+\frac{1}{2 \eta} \int_{\Omega}\left(\left|m_{N}^{\eta}\right|^{2}-1\right)^{2} \geq & \left\|\nabla m_{N}^{\eta}\right\|_{L^{2}(\Omega)}^{2}+\frac{1}{2}\left\|\frac{\partial u_{N}^{\eta}}{\partial t}\right\|_{L^{2}(\Omega)}^{2}+\frac{1}{4} \int_{\Omega}\left(\lambda^{\mathrm{e}}: \varepsilon\left(u_{N}^{\eta}\right)\right): \varepsilon\left(u_{N}^{\eta}\right) \\
& +\frac{1}{2} \int_{\Omega} \mathcal{Q}\left(m_{N}^{\eta}\right)+\frac{1}{2 \eta} \int_{\Omega}\left(\left|m_{N}^{\eta}\right|^{2}-1\right)^{2}-C\left\|m_{N}^{\eta}\right\|_{L^{4}(\Omega)}^{4} .
\end{aligned}
$$

Now we remark that $\left(|\xi|^{2}-1\right)^{2} \geq \frac{1}{2}|\xi|^{4}-1$ so

$$
C\left\|m_{N}^{\eta}\right\|_{L^{4}(\Omega)}^{4} \leq 2 C \int_{\Omega}\left(\left|m_{N}^{\eta}\right|^{2}-1\right)^{2}+2 C \operatorname{meas}(\Omega),
$$

and if $\eta$ is so that $2 C \leq \frac{1}{4 \eta}$, we obtain that

$$
\frac{1}{4 \eta} \int_{\Omega}\left(\left|m_{N}^{\eta}\right|^{2}-1\right)^{2}-C\left\|m_{N}^{\eta}\right\|_{L^{4}}^{4} \geq-2 C \operatorname{meas}(\Omega) .
$$

So if $\eta$ is small enough, we obtain that

$$
\begin{aligned}
\mathcal{E}_{N}^{\eta}+\frac{1}{2 \eta} \int_{\Omega}\left(\left|m_{N}^{\eta}\right|^{2}-1\right)^{2} \geq & \left\|\nabla m_{N}^{\eta}\right\|_{L^{2}(\Omega)}^{2}+\frac{1}{2}\left\|\frac{\partial u_{N}^{\eta}}{\partial t}\right\|_{L^{2}(\Omega)}^{2}+\frac{1}{4} \int_{\Omega}\left(\lambda^{\mathrm{e}}: \varepsilon\left(u_{N}^{\eta}\right)\right): \varepsilon\left(u_{N}^{\eta}\right) \\
& +\frac{1}{2} \int_{\Omega} \mathcal{Q}\left(m_{N}^{\eta}\right)+\frac{1}{4 \eta} \int_{\Omega}\left(\left|m_{N}^{\eta}\right|^{2}-1\right)^{2}-2 C \operatorname{meas}(\Omega) .
\end{aligned}
$$

Remark 7 The previous trick to absorb the bad sign term will be re-used in Part 3.

\subsection{Limit in the Galerkin Approximation}

From (16) together with the energy estimate (15), we obtain that for $\eta$ small enough

$$
\begin{array}{r}
\left\|\nabla m_{N}^{\eta}\right\|_{L^{2}(\Omega)}^{2}+\frac{1}{2}\left\|\frac{\partial u_{N}^{\eta}}{\partial t}\right\|_{L^{2}(\Omega)}^{2}+\frac{1}{4} \int_{\Omega}\left(\lambda^{\mathrm{e}}: \varepsilon\left(u_{N}^{\eta}\right)\right): \varepsilon\left(u_{N}^{\eta}\right)+\frac{1}{2} \int_{\Omega} \mathcal{Q}\left(m_{N}^{\eta}\right)+\frac{1}{4 \eta} \int_{\Omega}\left(\left|m_{N}^{\eta}\right|^{2}-1\right)^{2} \\
\leq 2 C \operatorname{meas}(\Omega)++\mathcal{E}_{N}^{\eta}(0)+\frac{1}{2 \eta} \int_{\Omega}\left(\left|P_{N}\left(m_{0}\right)\right|^{2}-1\right)^{2} .
\end{array}
$$

Claim: the right hand side of (17) is bounded uniformly with respect to $N$.

Proof of the claim: using Lemma 3, using that $|\mathcal{Q}(m)| \leq K|m|^{4}$ and the Sobolev embedding $H^{1}(\Omega) \subset L^{4}(\Omega)$, we get:

$$
\mathcal{E}_{N}^{\eta}(0) \leq C\left\|P_{N}\left(m_{0}\right)\right\|_{H^{1}(\Omega)}^{2}+\left\|\Pi_{N}\left(u_{1}\right)\right\|_{L^{2}(\Omega)}^{2}+C \int_{\Omega}\left(\lambda^{\mathrm{e}}: \mathcal{E}\left(\Pi_{N}\left(u_{0}\right)\right)\right): \varepsilon\left(\Pi_{N}\left(u_{0}\right)\right) .
$$

Since $P_{N}$ and $\Pi_{N}$ are orthogonal projection maps in $L^{2}$, we have:

$$
\left\|P_{N}\left(m_{0}\right)\right\|_{L^{2}(\Omega)} \leq\left\|m_{0}\right\|_{L^{2}(\Omega)} \text { and }\left\|\Pi_{N}\left(u_{1}\right)\right\|_{L^{2}(\Omega)} \leq\left\|u_{1}\right\|_{L^{2}(\Omega)} .
$$


Furthermore,

$$
\begin{aligned}
\left\|\nabla P_{N}\left(m_{0}\right)\right\|_{L^{2}(\Omega)}^{2} & =-\int_{\Omega} \Delta P_{N}\left(m_{0}\right) \cdot P_{N}\left(m_{0}\right) \\
& =-\int_{\Omega} \Delta P_{N}\left(m_{0}\right) \cdot m_{0} \\
& \text { since } V_{N} \text { is stable by } P_{N} \\
& =\int_{\Omega} \nabla P_{N}\left(m_{0}\right) \cdot \nabla m_{0} \\
\leq & \left\|\nabla P_{N}\left(m_{0}\right)\right\|_{L^{2}(\Omega)}\left\|\nabla m_{0}\right\|_{L^{2}(\Omega)} .
\end{aligned}
$$

So,

$$
\left\|\nabla P_{N}\left(m_{0}\right)\right\|_{L^{2}(\Omega)} \leq\left\|\nabla m_{0}\right\|_{L^{2}(\Omega)}
$$

In the same way,

$$
\begin{aligned}
\int_{\Omega}\left(\lambda^{\mathrm{e}}: \varepsilon\left(\Pi_{N}\left(u_{0}\right)\right)\right): \varepsilon\left(\Pi_{N}\left(u_{0}\right)\right) & =-\int_{\Omega} \operatorname{div}\left(\lambda^{\mathrm{e}}: \varepsilon\left(\Pi_{N}\left(u_{0}\right)\right)\right) \Pi_{N}\left(u_{0}\right) \\
& =-\int_{\Omega} \operatorname{div}\left(\lambda^{\mathrm{e}}: \varepsilon\left(\Pi_{N}\left(u_{0}\right)\right)\right) u_{0} \\
& \operatorname{since} W_{N} \text { is stable by } \operatorname{div}\left(\lambda^{\mathrm{e}}: \varepsilon\right) \\
= & \int_{\Omega}\left(\lambda^{\mathrm{e}}: \varepsilon\left(\Pi_{N}\left(u_{0}\right)\right)\right) \varepsilon\left(u_{0}\right) \\
\leq & \left(\int_{\Omega}\left(\lambda^{\mathrm{e}}: \varepsilon\left(\Pi_{N}\left(u_{0}\right)\right)\right) \varepsilon\left(\Pi_{N}\left(u_{0}\right)\right)\right)^{\frac{1}{2}}\left(\int_{\Omega}\left(\lambda^{\mathrm{e}}: \varepsilon\left(u_{0}\right)\right) \varepsilon\left(u_{0}\right)\right)^{\frac{1}{2}} \\
& \text { from } \operatorname{Lemma} 3 .
\end{aligned}
$$

So,

$$
\int_{\Omega}\left(\lambda^{\mathrm{e}}: \varepsilon\left(\Pi_{N}\left(u_{0}\right)\right)\right) \varepsilon\left(\Pi_{N}\left(u_{0}\right)\right) \leq \int_{\Omega}\left(\lambda^{\mathrm{e}}: \varepsilon\left(u_{0}\right)\right) \varepsilon\left(u_{0}\right) .
$$

We remark now that

$$
\begin{aligned}
\frac{1}{2 \eta} \int_{\Omega}\left(\left|P_{N}\left(m_{0}\right)\right|^{2}-1\right)^{2} \leq & \frac{1}{\eta}\left(1+\left\|P_{N}\left(m_{0}\right)\right\|_{L^{4}(\Omega)}^{4}\right) \\
\leq & C\left(1+\left\|m_{0}\right\|_{H^{1}(\Omega)}^{4}\right) \\
& \text { by Sobolev embeddings. }
\end{aligned}
$$

Inequalities (18), (19) and (20) yield that the right hand side of (17) is bounded uniformly with respect to $N$ and the proof of the claim is complete (we remark that at this step, the bound for the right hand side term depends on $\eta$ ).

Therefore we obtain for $\eta$ small enough an uniform bound for the following quantities:

- $\frac{\partial m_{N}^{\eta}}{\partial t}$ in $L^{2}\left(0, T_{N} ; L^{2}(\Omega)\right)$,

- $\nabla m_{N}^{\eta}$ in $L^{\infty}\left(0, T_{N} ; L^{2}(\Omega)\right)$,

- $m_{N}^{\eta}$ in $L^{\infty}\left(0, T_{N} ; L^{4}(\Omega)\right)$,

- $\nabla u_{N}^{\eta}$ in $L^{\infty}\left(0, T_{N} ; L^{2}(\Omega)\right)$,

- $\varepsilon\left(u_{N}^{\eta}\right)$ in $L^{\infty}\left(0, T_{N} ; L^{2}(\Omega)\right)$. 
This proves first that $T_{N}=+\infty$. In addition, since the bounds do not depend on $N$, we can assume by a diagonal extraction process that for all $T$, we have the following weak limits:

- $m_{N}^{\eta} \rightarrow m^{\eta}$ in $L^{\infty}\left(0, T ; H^{1}(\Omega)\right)$ weak *,

- $m_{N}^{\eta} \longrightarrow m^{\eta}$ in $L^{\infty}\left(0, T ; L^{4}(\Omega)\right)$ strong (with Lemma 4 ),

- $\frac{\partial m_{N}^{\eta}}{\partial t} \rightarrow \frac{\partial m^{\eta}}{\partial t}$ in $L^{2}\left(0, T ; L^{2}(\Omega)\right)$ weak,

- $u_{N}^{\eta} \rightarrow u^{\eta}$ in $L^{\infty}\left(0, T ; H^{1}(\Omega)\right)$ weak *,

- $\frac{\partial u_{N}^{\eta}}{\partial t} \rightarrow \frac{\partial m^{\eta}}{\partial t}$ in $L^{\infty}\left(0, T ; L^{2}(\Omega)\right)$ weak *.

So we can take the limit on the variational formulation of the Galerkin approximation (13) and in the energy formula (15) by convexity arguments.

Therefore we obtain for a fixed $\eta$ small enough that there exists $\left(m^{\eta}, u^{\eta}\right)$ weak solution of (12) and satisfying the energy formula for all $T$ :

$$
\mathcal{E}^{\eta}(T)+\frac{1}{2 \eta} \int_{\Omega}\left(\left|m^{\eta}\right|^{2}-1\right)^{2}+\int_{0}^{T} \int_{\Omega}\left|\frac{\partial m^{\eta}}{\partial t}\right|^{2}=\mathcal{E}^{\eta}(0) .
$$

where

$$
\begin{aligned}
\mathcal{E}^{\eta}(t)= & \int_{\Omega}\left(\left|\nabla m^{\eta}\right|^{2}+\Phi\left(m^{\eta}\right)+\frac{1}{2} \mathcal{Q}\left(m^{\eta}\right)-\varepsilon\left(u^{\eta}\right):\left(\lambda^{\mathrm{e}}:\left(\lambda^{\mathrm{m}}: m^{\eta} \otimes m^{\eta}\right)\right)\right) \\
& +\int_{\mathbb{R}^{3}}\left|h_{d}\left(m^{\eta}\right)\right|^{2}+\frac{1}{2} \int_{\Omega}\left(\left|\frac{\partial u^{\eta}}{\partial t}\right|^{2}+\left(\lambda^{\mathrm{e}}: \varepsilon\left(u^{\eta}\right)\right): \varepsilon\left(u^{\eta}\right)\right) .
\end{aligned}
$$

Remark 8 Since the initial data $m_{0}$ satisfies $\left|m_{0}\right|=1$ a.e., the right hand side of the energy estimate does not depend on $\eta$ since the penalization term vanishes at $t=0$. This is a crucial point to obtain uniform bound when $\eta$ tends to zero in the following section.

\section{Weak solutions for Landau-Lifschitz equation with magne- tostriction}

We take the limit in the penalized system when $\eta$ tends to zero. From the energy estimate (21) and from Remark 8, using the same arguments as in the previous section, we obtain that the following quantities are uniformly bounded with respect to $\eta$ :

- $\frac{\partial m^{\eta}}{\partial t}$ is bounded in $L^{2}\left(\mathbb{R}^{+} ; L^{2}(\Omega)\right)$,

- $\nabla m^{\eta}$ is bounded in $L^{\infty}\left(\mathbb{R}^{+} ; L^{2}(\Omega)\right)$,

- $m^{\eta}$ is bounded in $L^{\infty}\left(\mathbb{R}^{+} ; L^{4}(\Omega)\right)$,

- $\nabla u^{\eta}$ is bounded in $L^{\infty}\left(\mathbb{R}^{+} ; L^{2}(\Omega)\right)$,

- $\varepsilon\left(u^{\eta}\right)$ is bounded in $L^{\infty}\left(\mathbb{R}^{+} ; L^{2}(\Omega)\right)$.

With this bound, using the diagonal extraction process, we obtain that there exists $(m, u)$ such that for all $T$,

- $m^{\eta} \rightarrow m$ in $L^{\infty}\left(0, T ; H^{1}(\Omega)\right)$ weak *,

- $m^{\eta} \longrightarrow m$ in $L^{\infty}\left(0, T ; L^{4}(\Omega)\right)$ strong (with Lemma 4 ), 
- $\frac{\partial m^{\eta}}{\partial t} \rightarrow \frac{\partial m}{\partial t}$ in $L^{2}\left(0, T ; L^{2}(\Omega)\right)$ weak,

- $u^{\eta} \rightarrow u$ in $L^{\infty}\left(0, T ; H^{1}(\Omega)\right)$ weak *,

- $\frac{\partial u^{\eta}}{\partial t} \rightarrow \frac{\partial m}{\partial t}$ in $L^{\infty}\left(0, T ; L^{2}(\Omega)\right)$ weak *

Using (21), $\int_{\Omega}\left(\left|m^{\eta}\right|^{2}-1\right)^{2}$ tends to zero and since $m^{\eta} \longrightarrow m$ in $L^{\infty}\left(0, T ; L^{4}(\Omega)\right)$ strong, we obtain that

$$
|m|=1 \text { a.e. }
$$

In addition, using convexity or strong convergence arguments, taking the limit when $\eta$ tends to zero in (21) that for all $T$,

$$
\mathcal{E}(T)+\int_{0}^{T} \int_{\Omega}\left|\frac{\partial m}{\partial t}\right|^{2} \leq \mathcal{E}(0)
$$

where

$$
\begin{aligned}
\mathcal{E}(t)= & \int_{\Omega}\left(|\nabla m|^{2}+\Phi(m)+\frac{1}{2} \mathcal{Q}(m)-\varepsilon(u):\left(\lambda^{\mathrm{e}}:\left(\lambda^{\mathrm{m}}: m \otimes m\right)\right)+\frac{1}{2}\left(\lambda^{\mathrm{e}}: \varepsilon(u)\right): \varepsilon(u)\right) \\
& +\int_{\mathbb{R}^{3}}\left|h_{d}(m)\right|^{2}+\frac{1}{2} \int_{\Omega}\left|\frac{\partial u}{\partial t}\right|^{2} .
\end{aligned}
$$

In order to obtain that the weak limit $m$ satisfies the Landau-Lifschitz equation, as in [2] and in [12], we consider $\chi \in \mathcal{C}_{c}^{\infty}\left(\mathbb{R}^{+} ; \mathcal{C}^{\infty}(\Omega)\right)$ compactely supported in $[0, T[$, and we take the test function $(t, x) \mapsto m^{\eta}(t, x) \wedge \chi(t, x)$ in the weak formulation for the first equation of (12).

We obtain then that:

$$
\begin{aligned}
& \int_{0}^{T} \int_{\Omega}\left(\frac{\partial m^{\eta}}{\partial t}+m^{\eta} \wedge \frac{\partial m^{\eta}}{\partial t}\right) \cdot m^{\eta} \wedge \chi=-2 \int_{0}^{T} \int_{\Omega} \sum_{i=1}^{3} \frac{\partial m^{\eta}}{\partial x_{i}} \cdot \frac{\partial}{x_{i}}\left(m^{\eta} \wedge \chi\right) \\
& +2 \int_{0}^{T} \int_{\Omega}\left(h_{d}\left(m^{\eta}\right)+\Psi\left(m^{\eta}\right)+\left(\lambda^{\mathrm{m}}:\left(\lambda^{\mathrm{e}}: \varepsilon\left(u^{\eta}\right)\right)\right) m^{\eta}-\left(\lambda^{\mathrm{m}}:\left(\lambda^{\mathrm{e}}:\left(\lambda^{\mathrm{m}}: m^{\eta} \otimes m^{\eta}\right)\right)\right) m^{\eta}\right) \cdot\left(m^{\eta} \wedge \chi\right) .
\end{aligned}
$$

From algebraic calculations, we have:

$$
\begin{aligned}
\int_{0}^{T} \int_{\Omega}\left(\frac{\partial m^{\eta}}{\partial t}+m^{\eta} \wedge \frac{\partial m^{\eta}}{\partial t}\right) \cdot m^{\eta} \wedge \chi= & \int_{0}^{T} \int_{\Omega}\left(\frac{\partial m^{\eta}}{\partial t}-m^{\eta} \wedge \frac{\partial m^{\eta}}{\partial t}\right) \cdot \chi \\
& +\int_{0}^{T} \int_{\Omega}\left(\left|m^{\eta}\right|^{2}-1\right) \frac{\partial m^{\eta}}{\partial t} \cdot \chi-\int_{0}^{T} \int_{\Omega}\left(m^{\eta} \cdot \frac{\partial m^{\eta}}{\partial t}\right)\left(m^{\eta} \cdot \chi\right) .
\end{aligned}
$$

Since $m^{\eta} \longrightarrow m$ in $L^{\infty}\left(0, T ; L^{4}(\Omega)\right)$ strong and $\frac{\partial m^{\eta}}{\partial t} \rightarrow \frac{\partial m}{\partial t}$ in $L^{2}\left(0, T ; L^{2}(\Omega)\right)$ weak, we obtain that

$$
\begin{aligned}
\int_{0}^{T} \int_{\Omega}\left(\frac{\partial m^{\eta}}{\partial t}+m^{\eta} \wedge \frac{\partial m^{\eta}}{\partial t}\right) \cdot m^{\eta} \wedge \chi \longrightarrow & \int_{0}^{T} \int_{\Omega}\left(\frac{\partial m}{\partial t}-m \wedge \frac{\partial m}{\partial t}\right) \cdot \chi \\
& +\int_{0}^{T} \int_{\Omega}\left(|m|^{2}-1\right) \frac{\partial m}{\partial t} \cdot \chi-\int_{0}^{T} \int_{\Omega}\left(m \cdot \frac{\partial m}{\partial t}\right)(m \cdot \chi) .
\end{aligned}
$$

As $|m|=1$, we obtain that $m \cdot \frac{\partial m}{\partial t}=0$, and so

$$
\int_{0}^{T} \int_{\Omega}\left(\frac{\partial m^{\eta}}{\partial t}+m^{\eta} \wedge \frac{\partial m^{\eta}}{\partial t}\right) \cdot m^{\eta} \wedge \chi \longrightarrow \int_{0}^{T} \int_{\Omega}\left(\frac{\partial m}{\partial t}-m \wedge \frac{\partial m}{\partial t}\right) \cdot \chi .
$$


By the same kind of arguments, we take the limit in the right hand side of (23) and we obtain that

$$
\begin{aligned}
& \int_{\mathbb{R}^{+} \times \Omega}\left(\frac{\partial m}{\partial t}-m \wedge \frac{\partial m}{\partial t}\right) \chi(t, x) d t d x=2 \int_{\mathbb{R}^{+} \times \Omega} \sum_{i=1}^{3} m \wedge \frac{\partial m}{\partial x_{i}} \cdot \frac{\partial \chi}{\partial x_{i}}-2 \int_{\Omega}\left(h_{d}(m)+\Psi(m)\right) \cdot \chi \\
& -2 \int_{\Omega}\left(\left(\lambda^{\mathrm{m}}:\left(\lambda^{\mathrm{e}}: \varepsilon(u)\right)\right) m-\left(\lambda^{\mathrm{m}}:\left(\lambda^{\mathrm{e}}:\left(\lambda^{\mathrm{m}}: m \otimes m\right)\right)\right) m\right) \cdot \chi,
\end{aligned}
$$

By standart arguments, we pass to the limit in the wave equation, and we obtain that $(m, u)$ satisfies (5)-(6)-(7).

So we have proved the existence of a global in time weak solution of (5)-(6)-(7) satisfying the saturation constraint (1) and the energy estimate (22) and such that

- $m \in L^{\infty}\left(\mathbb{R}^{+} ; H^{1}(\Omega)\right)$,

- $\frac{\partial m}{\partial t} \in L^{2}\left(\mathbb{R}^{+} ; L^{2}(\Omega)\right)$,

- $u \in L^{\infty}\left(\mathbb{R}^{+} ; H^{1}(\Omega)\right)$

- $\frac{\partial u}{\partial t} \in L^{\infty}\left(\mathbb{R}^{+} ; L^{2}(\Omega)\right)$.

\section{$4 \omega$ limit set}

We fix a weak solution of (5)-(6)-(7) satisfying the previous conditions, so its $\omega$-limit set is non empty, that is we can consider $m_{\infty}$ such that there exists a sequence $\left(t_{n}\right)_{n}$ with $t_{n} \longrightarrow+\infty$ and such that $m\left(t_{n}\right) \rightarrow m_{\infty}$ in $H^{1}(\Omega)$ weakly and in $L^{p}(\Omega)$ strong for all $p<6$ by Sobolev theorems.

For a fixed $a>0$, we define $V^{n}(s, x)=m\left(t_{n}+s, x\right)$, defined on $]-a, a\left[\times \Omega\right.$ with values in $S^{2}$.

In the spirit of [12], we begin by performing the limit when $t_{n}$ tends to $+\infty$ for a fixed value of $a$. Using that $\frac{\partial m}{\partial t}$ is in $L^{2}\left(\mathbb{R}^{+} ; L^{2}(\Omega)\right)$, we obtain by this way the limit equation satisfied by $m_{\infty}$. This equation contains a terms $U_{\infty}$ coming from $u$. In order to obtain the limit equation satisfied by $U_{\infty}$, in a second step, we take the limit in the wave equation when $a$ tends to $+\infty$.

\subsection{Limit when $n$ tends to $+\infty$}

We remark that

$$
\frac{1}{2 a} \int_{-a}^{a} \int_{\Omega}\left|V^{n}(s, x)-m\left(t_{n}, x\right)\right|^{2} d x d s \leq \frac{1}{2 a} \int_{s=-a}^{a} \int_{x \in \Omega}\left|\int_{\tau=t_{n}}^{s} \frac{\partial m}{\partial t}(\tau, x) d \tau\right|^{2} d x d s \leq a \int_{t_{n}-a}^{+\infty} \int_{\Omega}\left|\frac{\partial m}{\partial t}\right|^{2},
$$

so extracting a subsequence if necessary, $V^{n} \longrightarrow m_{\infty}$ in $L^{2}(]-a, a\left[; L^{2}(\Omega)\right)$ strongly and almost everywhere, and by the bounds for the gradient, $V^{n} \rightarrow m_{\infty}$ in $L^{\infty}(]-a, a\left[; H^{1}(\Omega)\right)$ weak *.

In the same way, we define $U^{n}(s, x)=u\left(t_{n}+s, x\right)$. Let us introduce for $a>1$ the map $\rho_{a} \in \mathcal{C}^{\infty}(\mathbb{R} ; \mathbb{R})$ such that

$$
\left\{\begin{array}{l}
\rho_{a}(s)=0 \text { out of }[-a, a] \\
\rho_{a}(s)=1 \text { on }[-a+1, a-1] \\
0 \leq \rho_{a} \leq 1 \\
\left|\rho_{a}^{\prime}(s)\right| \leq 2
\end{array}\right.
$$

We set

$$
U_{a}^{n}=\frac{1}{2 a} \int_{-a}^{a} u\left(t_{n}+s, x\right) \rho_{a}(s) d s .
$$

By the estimates on $u$, there exists a constant $C$ such that for all $n$ and all $a,\left\|U_{a}^{n}\right\|_{H^{1}(\Omega)} \leq C$. 
Let $\xi \in H^{1}(\Omega)$ be a test function. In the weak formulation of (5) with the test function $\frac{1}{2 a} \rho_{a}\left(t-t_{n}\right) \xi(x)$, and we obtain that:

$$
\frac{1}{2 a} \int_{-a}^{a} \int_{\Omega}\left(\frac{\partial V^{n}}{\partial t}-V^{n} \wedge \frac{\partial V^{n}}{\partial t}\right) \rho_{a}(s) \xi(x) d s d x=T_{1}+\ldots+T_{4}
$$

where

$$
\begin{aligned}
& T_{1}=\frac{1}{a} \int_{-a}^{a} \int_{\Omega} \sum_{i} V^{n} \wedge \frac{\partial V^{n}}{\partial x_{i}} \cdot \frac{\partial \xi}{\partial x_{i}} \rho_{a}(s) d s d x, \\
& T_{2}=-\frac{1}{a} \int_{-a}^{a} \int_{\Omega} V^{n} \wedge\left(h_{d}\left(V^{n}\right)+\psi\left(V^{n}\right)\right) \cdot \xi(x) \rho_{a}(s) d s d x \\
& T_{3}=-\frac{1}{a} \int_{-a}^{a} \int_{\Omega} V^{n} \wedge\left(\lambda^{\mathrm{m}}:\left(\lambda^{\mathrm{e}}:\left(\varepsilon\left(U^{n}\right)\right)\right) V^{n}\right) \cdot \xi(x) \rho_{a}(s) d s d x, \\
& \left.T_{4}=\frac{1}{a} \int_{-a}^{a} \int_{\Omega} V^{n} \wedge\left(\left(\lambda^{\mathrm{m}}:\left(\lambda^{\mathrm{e}}:\left(\lambda^{\mathrm{m}}: V^{n} \otimes V^{n}\right)\right)\right) V^{n}\right) \xi(x)\right) \cdot \xi(x) \rho_{a}(s) d s d x .
\end{aligned}
$$

The left hand side term tends to zero when $n$ tends to $+\infty$, since

$$
\left|\frac{1}{2 a} \int_{-a}^{a} \int_{\Omega}\left(\frac{\partial V^{n}}{\partial t}-V^{n} \wedge \frac{\partial V^{n}}{\partial t}\right) \rho_{a}(s) \xi(x) d s d x\right| \leq \frac{2}{\sqrt{2 a}}\left(\int_{\left[t_{n}-a,+\infty\right]} \int_{\Omega}\left|\frac{\partial m}{\partial t}\right|^{2}\right)^{\frac{1}{2}}\|\xi\|_{L^{2}(\Omega)} .
$$

We denote by

$$
\overline{\rho_{a}}=\frac{1}{2 a} \int_{-a}^{a} \rho_{a}(s) d s
$$

From (24), we obtain that,

$$
T_{1} \longrightarrow 2 \overline{\rho_{a}} \int_{\Omega} \sum_{i=1}^{3} m_{\infty} \wedge \frac{\partial m_{\infty}}{\partial x_{i}} \cdot \frac{\partial \xi}{\partial x_{i}} d x \text { when } n \text { tends to }+\infty .
$$

Moreover, since $\psi$ is linear and since $h_{d}$ maps continuously $l^{2}(\Omega)$ in $L^{2}\left(\mathbb{R}^{3}\right)$, we have

$$
T_{2} \longrightarrow-2 \overline{\rho_{a}} \int_{\Omega} m_{\infty} \wedge\left(h_{d}\left(m_{\infty}\right)+\psi\left(m_{\infty}\right)\right) \xi(x) d x,
$$

where

Concerning $T_{4}$, we denote by $F\left(X_{1}, X_{2}, X_{3}, X_{4}\right)=X_{1} \wedge\left(\lambda^{\mathrm{m}}:\left(\lambda^{\mathrm{e}}:\left(\lambda^{\mathrm{m}}: X_{2} \otimes X_{3}\right)\right) X_{4}\right)$, so that

$$
T_{4}=\frac{1}{a} \int_{-a}^{a} \int_{\Omega} F\left(V^{n}, V^{n}, V^{n}, V^{n}\right) \cdot \xi(x) \rho_{a}(s) d s d x .
$$

By linearity we write $T_{4}$ on the following way:

$$
\begin{aligned}
T_{4}= & \frac{1}{a} \int_{-a}^{a} \int_{\Omega} F\left(V^{n}-m_{\infty}, V^{n}, V^{n}, V^{n}\right) \cdot \xi(x) \rho_{a}(s) d s d x \\
& +\frac{1}{a} \int_{-a}^{a} \int_{\Omega} F\left(m_{\infty}, V^{n}-m_{\infty}, V^{n}, V^{n}\right) \cdot \xi(x) \rho_{a}(s) d s d x \\
& +\frac{1}{a} \int_{-a}^{a} \int_{\Omega} F\left(m_{\infty}, m_{\infty}, V^{n}-m_{\infty}, V^{n}\right) \cdot \xi(x) \rho_{a}(s) d s d x \\
& +\frac{1}{a} \int_{-a}^{a} \int_{\Omega} F\left(m_{\infty}, m_{\infty}, m_{\infty}, V^{n}-m_{\infty}\right) \cdot \xi(x) \rho_{a}(s) d s d x \\
& +2 \overline{\rho_{a}} \int_{\Omega} F\left(m_{\infty}, m_{\infty}, m_{\infty}, m_{\infty}\right) \cdot \xi(x) d x .
\end{aligned}
$$


Since $V^{n}$ and $m_{\infty}$ are bounded by 1 in $L^{\infty}$, and since $V_{n} \longrightarrow m_{\infty}$ in $L^{2}$ strong, we obtain that

$$
T_{4} \longrightarrow 2 \overline{\rho_{a}} \int_{\Omega} F\left(m_{\infty}, m_{\infty}, m_{\infty}, m_{\infty}\right) \cdot \xi(x) d x .
$$

Concerning $T_{3}$, we denote by $G\left(X_{1}, X_{2}, Y\right)=X_{1} \wedge\left(\lambda^{\mathrm{m}}:\left(\lambda^{\mathrm{e}}: Y\right) X_{2}\right)$, so that

$$
T_{3}=-\frac{1}{a} \int_{-a}^{a} \int_{\Omega} G\left(V^{n}, V^{n}, \varepsilon\left(U^{n}\right)\right) \cdot \xi(x) \rho_{a}(s) d s d x .
$$

We have, in the spirit of the previous calculations,

$$
\begin{aligned}
T_{3}= & -\frac{1}{a} \int_{-a}^{a} \int_{\Omega} G\left(V^{n}-m_{\infty}, V^{n}, \varepsilon\left(U^{n}\right)\right) \cdot \xi(x) \rho_{a}(s) d s d x \\
& -\frac{1}{a} \int_{-a}^{a} \int_{\Omega} G\left(m_{\infty}, V^{n}-m_{\infty}, \varepsilon\left(U^{n}\right)\right) \cdot \xi(x) \rho_{a}(s) d s d x \\
& -\frac{1}{a} \int_{-a}^{a} \int_{\Omega} G\left(m_{\infty}, m_{\infty}, \varepsilon\left(U^{n}\right)\right) \cdot \xi(x) \rho_{a}(s) d s d x .
\end{aligned}
$$

The first two terms of the right hand side tend to zero when $n$ tends to $+\infty$, since $\varepsilon\left(U^{n}\right)$ is bounded in $L^{\infty}\left(\mathbb{R}^{+} ; L^{2}(\Omega)\right), V^{n}$ and $m_{\infty}$ are bounded by 1 in $L^{\infty}$, and since $V_{n} \longrightarrow m_{\infty}$ in $L^{2}$ strong.

The last term reads:

$$
-\frac{1}{a} \int_{-a}^{a} \int_{\Omega} G\left(m_{\infty}, m_{\infty}, \varepsilon\left(U^{n}\right)\right) \cdot \xi(x) \rho_{a}(s) d s d x=\int_{\Omega} G\left(m_{\infty}, m_{\infty}, \varepsilon\left(U_{a}^{n}\right)\right) \cdot \xi(x) d x
$$

so since $U_{a}^{n}$ is bounded in $H^{1}(\Omega)$ uniformly with respect to $n$ and $a$, extracting a subsequence, there exists a subsequence such that $U_{a}^{n} \rightarrow U_{a}$ in $H^{1}(\Omega)$ weak. Therefore,

$$
T_{3} \longrightarrow \int_{\Omega} G\left(m_{\infty}, m_{\infty}, \varepsilon\left(U_{a}\right)\right) \cdot \xi(x) d x \text { when } n \longrightarrow+\infty .
$$

At this step, we have proved that for all $a>1, m_{\infty}$ satisfies:

$$
\begin{array}{r}
\int_{\Omega} \sum_{i=1}^{3} m_{\infty} \wedge \frac{\partial m_{\infty}}{\partial x_{i}} \cdot \frac{\partial \xi}{\partial x_{i}} d x-\int_{\Omega} m_{\infty} \wedge\left(h_{d}\left(m_{\infty}\right)+\psi\left(m_{\infty}\right)+F\left(m_{\infty}, m_{\infty}, m_{\infty}, m_{\infty}\right)\right) \xi(x) d x \\
+\frac{1}{\overline{\rho_{a}}} \int_{\Omega} G\left(m_{\infty}, m_{\infty}, \varepsilon\left(U_{a}\right)\right) \cdot \xi(x) d x=0
\end{array}
$$

We take now the limit of this equation when $a$ tends to $+\infty$.

First, $\overline{\rho_{a}}$ tends to 1 . in addition, $U_{a}$ is uniformly bounded, so we can extract a subsequence such that $U_{a} \rightarrow U_{\infty}$ in $H^{1}(\Omega)$ weakly when $a$ tends to $+\infty$. Let us precise the equation satisfied by $U_{\infty}$.

We write the weak formulation of (6) taking the test function : $\xi(x) \rho_{a}\left(t_{n}+s\right)$. We obtain that:

$$
\begin{array}{r}
-\frac{1}{2 a} \int_{-a}^{a} \int_{\Omega} \frac{\partial U^{n}}{\partial t} \rho_{a}^{\prime}(s) \xi(x) d x d s+\frac{1}{2 a} \int_{-a}^{a} \int_{\Omega}\left(\lambda^{\mathrm{e}}: \varepsilon\left(U^{n}\right)\right): \varepsilon(\xi) \rho_{a}(s) d x d s \\
=\frac{1}{2 a} \int_{-a}^{a} \int_{\Omega}\left(\lambda^{\mathrm{e}}:\left(\lambda^{\mathrm{m}}: V^{n} \otimes V^{n}\right)\right): \varepsilon(\xi) \rho_{a}(s) d x d s
\end{array}
$$

When $n$ tends to $+\infty$, the right hand side term tends to

$$
\overline{\rho_{a}} \int_{\Omega}\left(\lambda^{\mathrm{e}}:\left(\lambda^{\mathrm{m}}: m_{\infty} \otimes m_{\infty}\right)\right): \varepsilon(\xi) d x
$$


The second term satisfies:

$$
\frac{1}{2 a} \int_{-a}^{a} \int_{\Omega}\left(\lambda^{\mathrm{e}}: \varepsilon\left(U^{n}\right)\right): \varepsilon(\xi) \rho_{a}(s) d s d x=\int_{\Omega}\left(\lambda^{\mathrm{e}}: \varepsilon\left(U_{a}^{n}\right)\right): \varepsilon(\xi) d x
$$

so it tends to $\int_{\Omega}\left(\lambda^{\mathrm{e}}: \varepsilon\left(U_{a}\right)\right): \varepsilon(\xi) d x$ when $n$ tends to $+\infty$, and after when $a$ tends to $+\infty$, the limit is $\int_{\Omega}\left(\lambda^{\mathrm{e}}: \varepsilon\left(U_{\infty}\right)\right): \varepsilon(\xi) d x$.

Concerning the first left hand side term, we estimate it on the following way:

$$
\begin{aligned}
\left|\frac{1}{2 a} \int_{-a}^{a} \int_{\Omega} \frac{\partial U^{n}}{\partial t} \rho_{a}^{\prime}(s) \xi(x) d x d s\right| & \leq \frac{1}{2 a} \int_{[-a,-a+1] \cup[a-1, a]} \int_{\Omega}\left|\frac{\partial U^{n}}{\partial t}\left\|\rho_{a}^{\prime}(s)\right\| \xi(x)\right| d x d s \\
& \leq \frac{1}{a} 4\|\xi\|_{L^{2}}\left\|\frac{\partial u}{\partial t}\right\|_{L^{\infty}\left(\mathbb{R}^{+} ; L^{2}(\Omega)\right)},
\end{aligned}
$$

so when $n$ tends to $+\infty$ and after when $a$ tends to $+\infty$, this term tends to zero.

Hence, $U_{\infty}$ satisfies:

$$
\int_{\Omega}\left(\lambda^{\mathrm{e}}: \varepsilon\left(U_{\infty}\right)\right): \varepsilon(\xi) d x=\int_{\Omega}\left(\lambda^{\mathrm{e}}:\left(\lambda^{\mathrm{m}}: m_{\infty} \otimes m \infty\right)\right): \varepsilon(\xi) d x .
$$

We have proved that if $m_{\infty}$ is in the $\omega$-limit set of a trajectory $m$, then is satisfies in the weak sense the following system:

$m_{\infty} \wedge\left(\Delta m_{\infty}+h_{d}\left(m_{\infty}\right)+\psi\left(m_{\infty}\right)+\left(\lambda^{\mathrm{m}}:\left(\lambda^{\mathrm{e}}: \varepsilon\left(u_{\infty}\right)\right)\right) m_{\infty}-\left(\lambda^{\mathrm{m}}:\left(\lambda^{\mathrm{e}}:\left(\lambda^{\mathrm{m}}: m_{\infty} \otimes m_{\infty}\right)\right)\right) m_{\infty}\right)=0$

where $u_{\infty}$ is deduced from $m_{\infty}$ by:

$$
\left\{\begin{array}{l}
u_{\infty} \in H_{0}^{1}(\Omega) \\
\operatorname{div}\left(\lambda^{\mathrm{e}}: \varepsilon\left(u_{\infty}\right)\right)=\operatorname{div}\left(\lambda^{\mathrm{e}}:\left(\lambda^{\mathrm{m}}: m_{\infty} \otimes m_{\infty}\right)\right) \text { in } \Omega
\end{array}\right.
$$

\section{References}

[1] F. Alouges, T. Rivière and S. Serfaty. Néel and cross-tie wall energies for planar micromagnetic configurations. A tribute to J. L. Lions. ESAIM Control Optim. Calc. Var. 8 (2002), 31-68.

[2] F. Alouges and A. Soyeur. On global weak solutions for Landau-Lifshitz equations: existence and nonuniqueness. Nonlinear Anal. 18 (1992), no. 11, 1071-1084.

[3] H. Ammari and K. Hamdache. Global existence and regularity of solutions to a system of nonlinear Maxwell equations. Journal of Mathematical Analysis and Applications 286 (2003), 51-63.

[4] H. Ammari, L. Halpern and K. Hamdache. Asymptotic analysis of thin ferromagnetic films. Asymptotic Analysis 24 (2000), 277-294.

[5] L. Ban̆as. Adaptative techniques for Landau-Lifschitz-Gilbert equation with magnetostriction. $J$. Comp. Appl. Math. 215 (2008), 304-310.

[6] L. Ban̆as, S. Bartels and A. Prohl. A convergent implicit finite element discretization of the MaxwellLandau-Lifshitz-Gilbert equation. SIAM J. Numer. Anal. 46 (2008), no. 3, 1399-1422.

[7] F. Boust, N. Vukadinovic and S. Labbé. 3D dynamic micromagnetic simulations of susceptibility spectra in soft ferromagnetic particles. ESAIM-Proc 22 (2008), 127-131. 
[8] F. Boyer and P. Fabrie. Eléments d'analyse pour l'étude de quelques modèles d'écoulements de fluides visqueux incompressibles. Mathématiques \& Applications 52, Springer-Verlag, Berlin, 2006.

[9] F. Brown. Micromagnetics. Wiley, New York (1963).

[10] G. Carbou. Stability of static walls for a three-dimensional model of ferromagnetic material. $J$. Math. Pures Appl. 93 (2010), 183-203

[11] G. Carbou, M. A. Efendiev, P. Fabrie. Relaxed model for the hysteresis in micromagnetism. Proc. Roy. Soc. Edinburgh Sect. A 139 (2009), no. 4, 759-773.

[12] G. Carbou and P. Fabrie. Time average in micromagnetism. J. Differential Equations 147 (1998), no. 2, 383-409.

[13] G. Carbou and P. Fabrie. Regular solutions for Landau-Lifschitz equation in a bounded domain. Differential Integral Equations 14 (2001), no. 2, 213-229.

[14] G. Carbou and P. Fabrie. Regular solutions for Landau-Lifschitz equation in $\mathbb{R}^{3}$. Commun. Appl. Anal. 5 (2001), no. 1, 17-30

[15] G. Carbou, P. Fabrie and O. Guès. On the ferromagnetism equations in the non static case. Comm. Pure Appli. Anal. 3 (2004), 367-393.

[16] G. Carbou and S. Labbé. Stability for static walls in ferromagnetic nanowires. Discrete Contin. Dyn. Syst. Ser. B 6 (2006), no. 2, 273-290.

[17] A. DeSimone, R. Kohn, S. Müller, F. Otto and R. Schäfer. Two-dimensional modelling of soft ferromagnetic films. R. Soc. Lond. Proc. Ser. A Math. Phys. Eng. Sci. 457 (2001), no. 2016, 29832991.

[18] L. Halpern and S. Labbé. Modélisation et simulation du comportement des matériaux ferromagétiques. Matapli 66 (2001), 70-86.

[19] S. Labbé. Fast computation for large magnetostatic systems adapted for micromagnetism. SISC SIAM J. on Sci. Comp. 26 (2005), no. 6, 2160-2175.

[20] S. Labbé and P.-Y. Bertin. Microwave polarisability of ferrite particles with non-uniform magnetization. Journal of Magnetism and Magnetic Materials 206 (1999), 93-105.

[21] L. Landau et E. Lifschitz, Electrodynamique des milieux continues, cours de physique théorique, tome VIII (ed. Mir) Moscou (1969).

[22] J. L. Lions and E. Magenes. Problèmes aux limites non homogènes et applications. Vol. 1. Travaux et Recherches Mathématiques, No. 17 Dunod, Paris 1968.

[23] A. Visintin. On Landau Lifschitz equation for ferromagnetism. Japan Journal of Applied Mathematics 1 (1985), no. 2, 69-84.

[24] H. Wynled. Ferromagnetism. Encyclopedia of Physics, Vol. XVIII / 2, Springer Verlag, Berlin (1966). 